\title{
Symmetry axioms and perceived ambiguity
}

\author{
Peter Klibanoff $^{1}$ - Sujoy Mukerji ${ }^{2}$ - Kyoungwon Seo $^{3}$
}

Received: 9 September 2016 / Accepted: 15 June 2017 / Published online: 4 July 2017

(C) The Author(s) 2017. This article is an open access publication

\begin{abstract}
Since at least de Finetti (Annales de l'Institut Henri Poincare 7:1-68, 1937), preference symmetry assumptions have played an important role in models of decision making under uncertainty. In the current paper, we explore (1) the relationship between the symmetry assumption of Klibanoff et al. (KMS) (Econometrica 82:1945-1978, 2014) and alternative symmetry assumptions in the literature, and (2) assuming symmetry, the relationship between the set of relevant measures, shown by KMS (2014) to reflect only perceived ambiguity, and the set of measures (which we will refer to as the Bewley set) developed by Ghirardato et al. (J Econ Theory 118:133-173, 2004), Nehring (Ambiguity in the context of probabilistic beliefs, working paper, 2001, Bernoulli without Bayes: a theory of utility-sophisticated preference, working paper, 2007) and Ghirardato and Siniscalchi (A more robust definition of multiple priors, working paper, 2007, Econometrica 80:2827-2847, 2012). This Bewley set is the main alternative offered in the literature as possibly representing perceived ambiguity. Regarding symmetry assumptions, we show that, under relatively mild conditions, a variety of preference symmetry conditions from the literature [including that in KMS (2014)] are equivalent. In KMS (2014), we showed that, under symmetry, the Bewley set and the set of relevant measures are not always the same. Here, we establish a preference condition, No Half Measures, that is necessary and sufficient for the two to be the same under symmetry.
\end{abstract}

We thank the referees and the Editor for helpful comments. Seo gratefully acknowledges the financial support of the National Science Foundation (SES-0918248) and KAIST.

Sujoy Mukerji

s.mukerji@qmul.ac.uk

Peter Klibanoff

peterk@kellogg.northwestern.edu

Kyoungwon Seo

seo8240@snu.ac.kr

1 Managerial Economics and Decision Sciences, Kellogg School of Management at Northwestern University, Evanston, IL, USA

2 School of Economics and Finance, Queen Mary University of London, London, UK

3 Business School, Seoul National University, Seoul, Korea 
This condition is rather stringent. Only when it is satisfied may the Bewley set be interpreted as reflecting only perceived ambiguity and not also taste aspects such as ambiguity aversion.

Keywords Symmetry · Beliefs · Ambiguity · Ambiguity aversion · Model uncertainty · Ellsberg

JEL Classifications D01 $\cdot$ D80 - D81 - D83

\section{Introduction}

Recent literature on ambiguity (meaning subjective uncertainty about probabilities over states) or model uncertainty (see e.g., surveys by Gilboa and Marinacci [17] and Marinacci [22]), makes heavy use of models of decision making under uncertainty. In this literature, a concern relevant to applying these models is how to connect the representation of preferences to perceived ambiguity or the models over which you are uncertain. In a recent contribution, Klibanoff, Mukerji and Seo (henceforth KMS) [21], we proposed an answer to this question for a broad class of symmetric preferences. In particular, we defined a notion of relevant measures and showed that these measures reflect only perceived ambiguity and not ambiguity aversion or risk aversion.

Since at least de Finetti [7], preference symmetry assumptions have played an important role in models of decision making under uncertainty. In the current paper we explore (1) the relationship between the symmetry assumption of KMS [21] and alternative symmetry assumptions in the literature, and (2) assuming symmetry, the relationship between the set of relevant measures developed by KMS [21] and the set of measures (which we will refer to as the Bewley set) developed by Ghirardato et al. [14], Nehring [24,25] and Ghirardato and Siniscalchi $[15,16]$. This latter relationship is of particular interest because this Bewley set is the main alternative offered in the literature as possibly representing perceived ambiguity. Regarding symmetry assumptions, we show that, under relatively mild conditions, a variety of preference symmetry conditions from the literature (including that in KMS [21]) are equivalent.

In KMS [21], we showed that, under symmetry, the Bewley set and the set of relevant measures are not always the same. This is important because, when they differ, the Bewley set is affected by changes in ambiguity aversion, and thus cannot be interpreted as reflecting only perceived ambiguity. In the present paper, we establish a preference condition, No Half Measures, that is necessary and sufficient for the two to be same for symmetric preferences. In contrast to the mild conditions used for the symmetry equivalence result, No Half Measures is rather stringent. Yet our results, combined with those in KMS [21], imply that it is only under these stringent conditions that the Bewley set may be interpreted as reflecting only perceived ambiguity and not also taste aspects such as ambiguity aversion.

When incorporating considerations of ambiguity or model uncertainty in finance models it is natural for the modeler to want to impose constraints on the preferences of the decision maker(s) in the model so that they reflect some type of calibration of perceived ambiguity to external data. For example, in an asset pricing model, the modeler may want to impose the restriction that an investor seeks to make her portfolio robust against only a certain limited set of stochastic processes or probabilitistic forecasts or different forecasting models (when incorporating model uncertainty) because these processes or forecasts pass certain tests of inference on past data. The theory developed in KMS [21] gives reasons why, if 
such constraints were to be imposed, one should do so through constraints on the relevant measures. KMS [21] shows where the relevant measures appear in representation functionals for symmetric versions of a variety of popular ambiguity models. For instance, in the $\alpha$-MEU model (see e.g., Ghirardato et al. [14]) and the smooth ambiguity model (see e.g., Klibanoff et al. [20], Nau [23], Seo [26]) and the extended MEU with contraction model (see e.g., Gajdos et al. [13]), the set of probabilistic forecast models entertained by the decision maker should be the set of measures that appear in the functional representations. Thus, reassuringly, the theory of relevant measures provides foundations to the common and intuitive practice in the way these ambiguity models are used.

However, KMS's theory [21] is predicated on a specification of symmetry. It is common to invoke some notion of symmetry/exchangeability, at least implicitly, to underpin statistical inference (e.g., of the kind used to justify restrictions on the set of forecasting models considered when incorporating model uncertainty). Our results in Sect. 3 demonstrate that KMS's specification of symmetry is not special and is in fact equivalent to notions invoked elsewhere in the literature under conditions that would be met in most settings.

Our study of the No Half Measures condition, in Sect. 4, shows that the possibilities of establishing foundations for practices concerning the way contraints on model uncertainty are incorporated in ambiguity models via theories invoking the Bewley set are limited. This is particularly true in the case of functional forms which allow for non-extreme attitudes to ambiguity that may be varied parametrically in the functional representation (such as in the $\alpha$-MEU model, the extended MEU with contraction model, and the smooth ambiguity model). For instance, in the $\alpha$-MEU model, the Bewley set can be identified with the set of measures appearing in the model only if $\alpha$ is 0 or 1 .

\section{Setting and preferences}

In this section, we describe aspects of the formal setting, notation and preferences from KMS [21] that will be useful here.

Let $S$ be a compact metric space and $\Omega=S^{\infty}$ the state space with generic element $\omega=\left(\omega_{1}, \omega_{2}, \ldots\right)$. The state space $\Omega$ is also compact metric (Aliprantis and Border [1, Theorems 2.61 and 3.36]). Denote by $\Sigma_{i}$ the Borel $\sigma$-algebra on the $i$-th copy of $S$, and by $\Sigma$ the product $\sigma$-algebra on $S^{\infty}$. An act is a simple Anscombe-Aumann act, a measurable $f: S^{\infty} \rightarrow X$ having finite range (i.e., $f\left(S^{\infty}\right)$ is finite) where $X$ is the set of lotteries (i.e., finite support probability measures on an outcome space $Z$ ). The set of acts is denoted by $\mathcal{F}$, and $\succsim$ is a binary relation on $\mathcal{F} \times \mathcal{F}$. As usual, we identify a constant act (an act yielding the same element of $X$ on all of $S^{\infty}$ ) with the element of $X$ it yields.

Denote by $\Pi$ the set of all finite permutations on $\{1,2, \ldots\}$ i.e., all one-to-one and onto functions $\pi:\{1,2, \ldots\} \rightarrow\{1,2, \ldots\}$ such that $\pi(i)=i$ for all but finitely many $i \in$ $\{1,2, \ldots\}$. For $\pi \in \Pi$, let $\pi \omega=\left(\omega_{\pi(1)}, \omega_{\pi(2)}, \ldots\right)$ and $(\pi f)(\omega)=f(\pi \omega)$.

For any topological space $Y, \Delta(Y)$ denotes the set of (countably additive) Borel probability measures on $Y$. Unless stated otherwise, a measure is understood as a countably additive Borel measure. For later use, $b a(Y)$ is the set of finitely additive bounded real-valued set functions on $Y$, and $b a_{+}^{1}(Y)$ the set of non-negative probability charges in $b a(Y)$. A measure $p \in \Delta\left(S^{\infty}\right)$ is called symmetric if the order doesn't matter, i.e., $p(A)=p(\pi A)$ for all $\pi \in \Pi$, where $\pi A=\{\pi \omega: \omega \in A\}$. Denote by $\ell^{\infty}$ the i.i.d. measure with the marginal $\ell \in \Delta(S)$. Define $\int_{S^{\infty}} f d p \in X$ by $\left(\int_{S^{\infty}} f d p\right)(B)=\left(\int_{S^{\infty}} f(\omega)(B) d p(\omega)\right)$. (Since $f$ is simple, this is well-defined.) 
Fix $x_{*}, x^{*} \in X$ such that $x^{*} \succ x_{*}$. For any event $A \in \Sigma, 1_{A}$ denotes the act giving $x^{*}$ on $A$ and $x_{*}$ otherwise. Informally, this is a bet on $A$. More generally, for $x, y \in X, x A y$ denotes the act giving $x$ on $A$ and $y$ otherwise. A finite cylinder event $A \in \Sigma$ is any event of the form $\left\{\omega: \omega_{i} \in A_{i}\right.$ for $\left.i=1, \ldots, n\right\}$ for $A_{i} \in \Sigma_{i}$ and some finite $n$.

Endow $\Delta(S), \Delta(\Delta(S))$ and $\Delta\left(S^{\infty}\right)$ with the relative weak* topology. To see what this is, consider, for example, $\Delta(S)$. The relative weak* topology on $\Delta(S)$ is the collection of sets $V \cap \Delta(S)$ for weak* open $V \subseteq b a(S)$, where the weak* topology on $b a(S)$ is the weakest topology for which all functions $\ell \longmapsto \int \psi d \ell$ are continuous for all bounded measurable $\psi$ on $S$. Also note that a net $\ell_{\alpha} \in b a(S)$ converges to $\ell \in b a(S)$ under the weak* topology if and only if $\int \psi d \ell_{\alpha} \rightarrow \int \psi d \ell$ for all bounded measurable $\psi$ on $S$. For a set $D \subseteq \Delta(S)$, denote the closure of $D$ in the relative weak* topology by $\bar{D}$.

The support of a probability measure $m \in \Delta(\Delta(S))$, denoted supp $m$, is a relative weak* closed set such that $m\left((\operatorname{supp} m)^{c}\right)=0$ and if $L \cap \operatorname{supp} m \neq \emptyset$ for relative weak* open $L$, $m(L \cap \operatorname{supp} m)>0$. (See e.g., Aliprantis and Border [1, p.441].)

Let $\Psi_{n}(\omega) \in \Delta(S)$ denote the empirical frequency operator $\Psi_{n}(\omega)(A)=\frac{1}{n} \sum_{t=1}^{n} I$ $\left(\omega_{t} \in A\right)$ for each event $A$ in $S$. Define the limiting frequency operator $\Psi$ by $\Psi(\omega)(A)=$ $\lim _{n} \Psi_{n}(\omega)(A)$ if the limit exists and 0 otherwise. Also, to map given limiting frequencies or sets of limiting frequencies to events in $S^{\infty}$, we consider the natural inverses $\Psi^{-1}(\ell)=$ $\{\omega: \Psi(\omega)=\ell\}$ and $\Psi^{-1}(L)=\{\omega: \Psi(\omega) \in L\}$ for $\ell \in \Delta(S)$ and $L \subseteq \Delta(S)$.

The following axioms are used in describing assumptions on preferences $\succsim$ at various points in this paper. For remarks and interpretation of these axioms see KMS [21].

Axiom 1 (C-complete Preorder) $\succsim$ is reflexive, transitive and the restriction of $\succsim$ to $X$ is complete.

Axiom 2 (Monotonicity) If $f(\omega) \succsim g(\omega)$ for all $\omega \in S^{\infty}, f \succsim g$.

Axiom 3 (Risk Independence) For all $x, x^{\prime}, x^{\prime \prime} \in X$ and $\alpha \in(0,1), x \succsim x^{\prime}$ if and only if $\alpha x+(1-\alpha) x^{\prime \prime} \succsim \alpha x^{\prime}+(1-\alpha) x^{\prime \prime}$.

Axiom 4 (Non-triviality) There exist $x, y \in X$ such that $x>y$.

The key axiom is Event Symmetry which implies that the ordinates of $S^{\infty}$ are viewed as interchangeable.

Axiom 5 (Event Symmetry) For all finite cylinder events $A \in \Sigma$ and finite permutations $\pi \in \Pi$,

$$
\alpha 1_{A}+(1-\alpha) h \sim \alpha 1_{\pi A}+(1-\alpha) h \text { for all } \alpha \in[0,1] \text { and all acts } h \in \mathcal{F} .
$$

We now state three forms of continuity that are used in the paper.

Axiom 6 (Mixture Continuity) For all $f, g, h \in \mathcal{F}$, the sets $\{\lambda \in[0,1]: \lambda f+(1-\lambda) g \succsim h\}$ and $\{\lambda \in[0,1]: h \succsim \lambda f+(1-\lambda) g\}$ are closed in $[0,1]$.

To describe our second and third continuity axioms, it is notationally convenient to introduce the following binary relation ${ }^{*}$ (see e.g., Ghirardato et al. [14]) derived from $\succsim$ :

$$
f \succsim^{*} g \text { if } \alpha f+(1-\alpha) h \succsim \alpha g+(1-\alpha) h \text { for all } \alpha \in[0,1] \text { and } h \in \mathcal{F} .
$$

A weakening of Mixture Continuity of $\succsim$ that is sufficient for some of our results is

Axiom 7 (Mixture Continuity of $\succsim^{*}$ ) For all $f, g, h \in \mathcal{F}$, the sets $\{\lambda \in[0,1]: \lambda f+$ $\left.(1-\lambda) g \succsim^{*} h\right\}$ and $\left\{\lambda \in[0,1]: h \succsim^{*} \lambda f+(1-\lambda) g\right\}$ are closed in $[0,1]$. 
Finally, borrowed from Ghirardato et al. [14], we have the following adaptation of Arrow's [3] monotone continuity:

Axiom 8 (Monotone Continuity of $\succsim^{*}$ ) For all $x, x^{\prime}, x^{\prime \prime} \in X$, if $A_{n} \searrow \varnothing$ and $x^{\prime} \succ x^{\prime \prime}$, then $x^{\prime} \succsim^{*} x A_{n} x^{\prime \prime}$ for some $n$.

Definition $2.1 \succsim$ satisfies Weak Continuous Symmetry if it satisfies all of the above axioms except possibly Mixture Continuity.

When we say that $\succsim$ is Weak Continuous Symmetric, we mean that it satisfies Weak Continuous Symmetry.

\section{Relating Event Symmetry to the literature}

A key axiom in our approach is Event Symmetry. We now show that this condition relates quite closely to a variety of other conditions from the literature, including strengthenings of de Finetti [7]'s Exchangeability, of Hewitt and Savage [19]'s Symmetry, of Seo [26]'s Dominance and of Klibanoff et al. [20]'s Consistency. One of those conditions (condition (viii) below) requires some additional definitions which we state next.

Definition 3.1 For $f \in \mathcal{F}, f^{\Psi}$ is the (not necessarily simple) act uniquely defined as follows:

$$
f^{\Psi}(\omega)= \begin{cases}\int_{S^{\infty}} f d \ell^{\infty} & \text { if } \ell=\Psi(\omega) \in \Delta(S) \\ \delta_{x^{*}} & \{\omega: \Psi(\omega) \text { is not defined. }\}\end{cases}
$$

Note this definition associates with each act $f$ an act $f^{\Psi}$ that, for each event $\{\omega: \Psi(\omega)=\ell\}$ corresponding to the limiting frequencies generated by $\ell$, yields the lottery generated by $f$ under the assumption that the i.i.d. process $\ell^{\infty}$ governs the realization of the state.

Since $f^{\Psi}$ need not be simple, but is an element of the space $\hat{\mathcal{F}}$ of all bounded and measurable functions from $\Omega$ to $X$, it is necessary to consider extending $\succsim$ to $\hat{\mathcal{F}}$. In particular, we consider extensions continuous in the following sense: $\hat{\succsim}$ on $\hat{\mathcal{F}}$ satisfies Norm Continuity if $f \hat{\succsim} g$ whenever $f_{k} \hat{\succsim} g_{k}$ for all $k=1,2, \ldots$ and $f_{k}$ and $g_{k}$ norm-converge to $f$ and $g$ respectively.

We now show that, under mild conditions, Event Symmetry is in fact equivalent to a variety of other conditions appearing in the literature. A first contribution, then, is to show that despite the seeming variety of preference formalizations of symmetry in the ambiguity literature, reassuringly their differences will not matter in most settings. Secondly, this also shows that results from KMS [21] derived assuming Event Symmetry are robust in the sense that they would continue to hold under alternative symmetry formulations.

Theorem 3.1 The following conditions are equivalent under the assumption that $\succsim$ is reflexive, transitive and satisfies Mixture Continuity:

(i) for every $f \in \mathcal{F}$ and $\pi \in \Pi, f \sim \frac{1}{2} f+\frac{1}{2} \pi f$,

(ii) for every $f \in \mathcal{F}, \pi \in \Pi$ and $\alpha \in[0,1], f \sim \alpha \pi f+(1-\alpha) f$,

(iii) for every $f \in \mathcal{F}$ and $\pi_{i} \in \Pi, f \sim \frac{1}{n} \sum_{i=1}^{n} \pi_{i} f$,

(iv) for every $f \in \mathcal{F}, \pi_{i} \in \Pi$ and $\alpha_{i} \in[0,1]$ with $\sum_{i=1}^{n} \alpha_{i}=1, f \sim \sum_{i=1}^{n} \alpha_{i} \pi_{i} f$, and

(v) for every $f \in \mathcal{F}$ and $\pi \in \Pi, f \sim^{*} \pi f$.

Moreover, the above are equivalent to each of the following under the assumption that $\succsim$ satisfies C-complete Preorder, Mixture Continuity, Monotonicity, Risk Independence, Non-triviality and Monotone Continuity of $\succsim^{*}$ : 
(vi) Event Symmetry,

(vii) for every $f, g \in \mathcal{F}$, if $\int f d p \succsim \int g d p$ for all symmetric $p \in \Delta\left(S^{\infty}\right)$, then $f \succsim g$. Finally, if, in addition, there exists an extension of $\succsim$ to $\hat{\mathcal{F}}$ that is reflexive, transitive and satisfies Norm Continuity, then the following is equivalent to all of the above:

(viii) for $f, g \in \mathcal{F}, f \succsim g$ if and only if $f^{\Psi} \hat{\succsim} g^{\Psi}$, if $\hat{\succsim}$ is any such extension.

All of these conditions are strengthenings of Hewitt and Savage [19]'s symmetry: given $p \in \Delta\left(S^{\infty}\right), p(A)=p(\pi A)$ for all finite cylinder events $A \in \Sigma$. In terms of preference, this translates into $1_{A} \sim 1_{\pi A}$ for all such events and all permutations $\pi \in \Pi{ }^{1}$ Event Symmetry strengthens this by requiring the indifference to be preserved under mixture with any common third act. Under reflexivity, transitivity and Mixture Continuity of $\succsim$, conditions (i)-(v) each imply Event Symmetry.

Condition (ii) is closely related to Epstein and Seo [10]'s Strong Exchangeability, the first behavioral axiom in the literature that captures the idea that the agent views all experiments as identical, i.e., i.i.d. (See Epstein and Seo [10] for a behavioral interpretation of condition (ii). A similar interpretation applies to (i), (iii) and (iv).) Their axiom states that condition (ii) holds when $f$ depends only on a finite number of experiments. However, under their regularity axiom, their Strong Exchangeability extends to every act $f$ and hence is equivalent to condition (ii).

Epstein and Seo [10], under MEU, characterize two models of preferences where the decision maker is indifferent to permutations - one that restricts to identical experiments (no ambiguity on idiosyncratic factors) and permits ambiguity about parameters, and the other that allows ambiguity on idiosyncratic factors but rules out ambiguity about parameters. Only the former of the two models satisfies (ii). Epstein and Seo [12] go further and characterize a single model within MEU that reflects ambiguity on both at the same time. The unifying framework permits a behavioral distinction between idiosyncratic factors and parameters as two sources of ambiguity.

Condition (i) is a special case of condition (ii) when $\alpha=\frac{1}{2}$. Since Epstein and Seo [10] consider MEU models, they could restrict to the case $\alpha=\frac{1}{2}$. The above theorem shows that $\alpha=\frac{1}{2}$ is sufficient to capture the same idea in general as long as reflexivity, transitivity and Mixture Continuity of $\succsim$ hold.

De Castro and Al-Najjar [2,5] use condition (iii) and its generalization to collections of transformations $\Gamma$ other than the finite permutations $\Pi$. They provide conditions on $\Gamma$ under which complete, transitive, monotonic, continuous and risk independent preferences satisfying (iii) with respect to $\Gamma$ are such that each act is indifferent to an associated act based on limiting frequencies (where the notion of limiting frequencies uses the given $\Gamma$ ) where the associated act is constructed much like $f^{\Psi}$ above with $\Gamma$-ergodic measures replacing the i.i.d. measures as parameters. They also prove a representation theorem for a utility function similar to (4.2) using an expected utility assumption on parameter-based acts added to the conditions on preferences and on $\Gamma$ mentioned in the previous sentence.

Condition (iv) strengthens condition (iii).

Condition (v) is stronger than Event Symmetry in that $f$ is not necessarily a binary act.

Condition (vii) is analogous to Seo [26]'s Dominance and Cerreia-Vioglio et al. [6]'s Consistency, and condition (viii) to Klibanoff et al. [20]'s Consistency. Seo's Dominance is stated with lotteries over acts, objects that are not available in the domain of this paper, and condition (vii) restricts $p \in \Delta\left(S^{\infty}\right)$ to be symmetric while $p$ is unrestricted in Seo's

${ }^{1}$ Epstein and Seo [11] assume $f \sim \pi f$ for all permutations $\pi$ and acts $f$. They show that there is a modeling trade-off between this symmetry axiom, a type of dynamic consistency and ambiguity. 
Dominance. Thus, the difference between the two is that Seo's Dominance requires $f$ to induce a better lottery than $g$ under all processes, not just symmetric ones. The reason for the additional restriction here is to reflect the fact that the experiments are symmetric. We want to include as reflecting dominance, for example, the following case:

$$
f(\omega)=x^{*} H_{1} x_{*} \text { and } g(\omega)=\left(\frac{1}{2} x^{*}+\frac{1}{2} x_{*}\right) H_{2} x_{*}
$$

where $H_{i}=\left\{\omega \in S^{\infty}: \omega_{i}=H\right\}$ and $S=\{H, T\}$. The act $f$ is a bet that the first coin comes up heads, and the act $g$ is a bet that the second coin comes up heads but with a less valuable reward for winning. Under symmetry of the experiments, it is intuitively clear that $f$ is better than $g$. Condition (vii) indeed implies that $f \succsim g$, while Seo's Dominance would not - for example, when $p=\delta_{T H T T T} . ., \int g d p \succ \int f d p$.

Cerreia-Vioglio et al. [6]'s Consistency is similar to condition (vii), but instead of considering transformations (e.g. permutations) and/or all symmetric measures, they assume a family of objectively rational probability measures on a general state space and require $\int f d p \succsim \int g d p$ for all measures $p$ in that family. Taking the family to be all symmetric measures makes Cerreia-Vioglio et al.'s Consistency exactly condition (vii). With their Consistency they provide representation theorems for Bewley preference, Choquet expected utility, variational preferences, uncertainty averse preferences and the smooth ambiguity model. Also, they prove that the generalization of condition (v) to other collections of transformations $\Gamma$ together with some (mild) axioms imply their Consistency when the family of objectively rational measures is the $\Gamma$-invariant measures.

The content of condition (viii) is that given the "knowledge" that everything is driven by some (as yet unknown) i.i.d. process, it seems reasonable that when evaluating an act, the individual would ultimately care only about the induced mapping from the space of i.i.d. processes to the lotteries generated under each process. Klibanoff et al. [20]'s Consistency assumption says that when evaluating an act, an individual cares only about the induced mapping from probability measures on the state space to lotteries. Their assumption was stated in terms of acts and "second order acts" (maps from probability measures on the state space to outcomes). The latter objects do not appear as such in the present paper, but their role is played by the subset of acts measurable with respect to limiting frequency events, the acts

$f^{\Psi} \in \hat{\mathcal{F}}$. The identification (in terms of preference) of $f$ with $f^{\Psi}$ stated in condition (viii) is analogous to the identification of $f$ with an "associated second order act" in Klibanoff et al. [20]'s Consistency with the qualification that $f^{\Psi}$ only induces the same mapping from probability measures to lotteries as $f$ for i.i.d. probability measures. Thus, condition (viii) strengthens Klibanoff et al. [20]'s Consistency to incorporate the known symmetry of the ordinates in the same way that condition (vii) strengthened Seo [26]'s Dominance. Theorem 3.1 says that, under our other axioms, each of these strengthenings is equivalent to Event Symmetry. One can view the equivalence of condition (iii) and condition (viii) as following from the i.i.d. case of the sufficient statistic result in Al-Najjar and de Castro [2].

\section{When do relevant measures and the Bewley set agree?}

We begin by recalling definitions of these sets and some key results from KMS [21] that we build upon.

KMS [21] defined what it means for a marginal $\ell \in \Delta(S)$ to be a relevant measure. For notational convenience, let $\mathcal{O}_{\ell}$ be the collection of open subsets of $\Delta(S)$ that contains $\ell$. That is, for $\ell \in \Delta(S), \mathcal{O}_{\ell}=\{L \subseteq \Delta(S): L$ is open, $\ell \in L\}$. 
Definition 4.1 A measure $\ell \in \Delta(S)$ is relevant (according to preferences $\succsim$ ) if, for any $L \in \mathcal{O}_{\ell}$, there are $f, g \in \mathcal{F}$ such that $f \nsim g$ and $\int f d \hat{\ell}^{\infty}=\int g d \hat{\ell}^{\infty}$ for all $\widehat{\imath} \in \Delta(S) \backslash L$.

In words, $\ell$ is relevant if it satisfies the following property: For each open set containing $\ell$, there are acts that are not indifferent despite generating identical induced distributions over outcomes when any measure outside this set governs the independent realization of each ordinate $S$. From KMS [21], we know that the set of relevant measures, $R$, is closed and that, a measure is not relevant if and only if the limiting frequency event generated by some open neighborhood of that measure is null according to the preferences. Thus, behavior is as if relevant measures were the possible resolutions of ambiguity. In this sense, the relevant measures reflect perceived ambiguity. Furthermore KMS [21, Theorem 3.4] showed that, under mild conditions, the set of relevant measures reflects only perceived ambiguity. Notably, the set is not affected by increases or decreases in ambiguity aversion.

The Bewley set, $C$, is defined using a Bewley-style (Bewley [4]) representation of the induced relation $\succsim^{*}$ (defined by (2.2)). For Weak Continuous Symmetric preferences violating Anscombe-Aumann Independence, $\succsim^{*}$ is incomplete relative to $\succsim$. In the context of Weak Continuous Symmetric preferences, KMS [21, Theorem 4.5] provided a Bewley-style (Bewley [4]) representation result for $\succsim^{*}$, identifying both the Bewley set $C$ and the set of relevant measures $R$. It is of interest to note that this result is a generalization of de Finetti's theorem. We restate it here, and will make use of it in proving several of the results of this section:

Theorem $4.1 \succsim$ is Weak Continuous Symmetric if and only if $\succsim$ is transitive and there exist a non-empty compact convex set $M \subseteq \Delta(\Delta(S))$ and a non-constant $v N M$ utility function $u$ such that

$$
f \succsim^{*} g \text { if and only if } \int u(f) d p \geq \int u(g) d p \text { for all } p \in C,
$$

where $C=\left\{\int \ell^{\infty} d m(\ell): m \in M\right\}$. Furthermore $R=\overline{\bigcup_{m \in M} \operatorname{supp} m}$ and $M$ is unique.

The main contribution of this section is to show that preference condition No Half Measures, defined below, is equivalent to the set of relevant measures fully determining the Bewley set, and thus necessary and sufficient for the Bewley set to inherit the interpretation of reflecting only perceived ambiguity from the set of relevant measures.

First a preliminary definition: Let $\Sigma^{\Psi}$ be the $\sigma$-algebra generated by the sets

$$
\Psi^{-1}(\ell) \text { for } \ell \in \Delta(S) \text {. }
$$

This algebra contains events based on limiting frequencies.

Condition (No Half Measures) For $A \in \Sigma^{\Psi}$, at least one of the following holds:

(i) If $1_{S^{\infty}} \succ x \in X$, then $\alpha x+(1-\alpha) f \succsim \alpha 1_{A}+(1-\alpha) f$ for some $f \in \mathcal{F}$ and $\alpha \in(0,1]$;

(ii) For all $f \in \mathcal{F}$ and $\alpha \in(0,1], \alpha 1_{\emptyset}+(1-\alpha) f \sim \alpha 1_{A}+(1-\alpha) f$.

To understand what this condition says, fix any bet on an event $A \in \Sigma^{\Psi}$ and let $x^{*} \in X$ be the lottery you get if you win and $x_{*}$ the lottery if you lose (with $x^{*} \succ x_{*}$ ). Imagine agreeing to lose for sure (i.e., having the bet shrunk from $A$ to the empty set) in return for improvement in the losing lottery (call the new losing lottery $w$, with $x^{*} \succ w \succsim x_{*}$ ). Notice that this is a tradeoff between utility on $A$ and $A^{c}$ - on $A, x^{*}$ is lowered to $w$, while on $A^{c}, x_{*}$ is raised to $w$. No Half Measures says that either there is always some mixture within which you do not prefer to make this trade-off (i.e., sometimes the increase on $A^{c}$ is worth less than any reduction 
on $A$ ), or, for every mixture, you are willing to agree to substitute a sure loss without any improvement in the losing stakes (i.e., you require no compensation for the reduced payoff on $A$ ). When this is true, then identifying the measures $\ell$ that are relevant is the same as identifying the measures that sometimes have an infinite marginal rate of substitution (i.e., that get assigned full belief). ${ }^{2}$ Thus, under this condition, classifying measures into those that sometimes get positive weight and those that do not is the same as classifying measures according to the range of marginal rates of substitution they sometimes receive. When this condition fails, the latter classification will make use of variations in ranges of marginal rates of substitution, not just zero vs. positive. Intuitively, these intermediate marginal rates of substitution depend not only on perceived ambiguity, but also on taste aspects of preferences such as ambiguity aversion.

We now show the connection between No Half Measures and the relation between the Bewley set and the set of relevant measures.

Theorem 4.2 Suppose $\succsim$ is Weak Continuous Symmetric. Then $\succsim$ satisfies No Half Measures if and only if $C=\left\{\int \ell^{\infty} d m(\ell): m \in \Delta(R)\right\}$ in (4.1).

Recall that, without No Half Measures, $C=\left\{\int \ell^{\infty} d m(\ell): m \in M\right\}$ where $M \subseteq \Delta(R)$, so that knowing $R$ need not pin down $C$. Our theorem shows that the No Half Measures condition is identifying those cases in which $C$ reflects only the set $R$ of processes considered relevant. In this sense, No Half Measures is required for the approach in Ghirardato et al. [14], Nehring [24] and Ghirardato and Siniscalchi $[15,16]$ based on $C$ to agree with the relevant measures and thus be interpretable as reflecting only perceived ambiguity and not perceived ambiguity intertwined with ambiguity aversion.

How restrictive is the No Half Measures condition?

One restriction is that No Half Measures implies there is only a finite set of relevant measures, and so only a finite number of possible resolutions of ambiguity.

Theorem 4.3 If a Weak Continuous Symmetric $\succsim$ satisfies No Half Measures then R is finite.

What are the further implications of No Half Measures in the context of specific decision models? We begin with the Maxmin expected utility (MEU) model (Gilboa and Schmeidler [18]). For this model, No Half Measures implies the set of measures in the representation are the i.i.d. products generated by the measures in $R$. We then examine the $\alpha$-MEU model and show there, when $R$ is non-singleton No Half Measures is only compatible with extreme values of $\alpha$ ( $\alpha$ equals 0 or 1 ). Similarly, we conclude this subsection with an examination of the smooth ambiguity model-when $R$ is non-singleton we show No Half Measures places non-trivial restrictions on the function $\phi .^{3}$

\subsection{MEU model}

Theorem 4.4 Suppose there is a non-constant $v N-M$ utility function $u$, and a non-empty compact convex set $M \subseteq \Delta(\Delta(S))$ such that, for $F \equiv\left\{\int \ell^{\infty} d m(\ell): m \in M\right\}$,

$$
V(f) \equiv \min _{p \in F} \int u(f) d p
$$

\footnotetext{
${ }^{2}$ A measure that always gets less than full belief will not be relevant, which motivates the name, No Half Measures.

${ }^{3}$ Similarly, though we do not fully investigate it, No Half Measures imposed on the Vector expected utility model (Siniscalchi [28]) will imply non-trivial restrictions on the adjustment function $A$ and the adjustment factors $\zeta_{i}$ appearing in that model.
} 
represents $\succsim$. Then $\succsim$ satisfies No Half Measures if and only if

$$
V(f)=\min _{\ell \in R} \int_{S^{\infty}} u(f) d \ell^{\infty}
$$

where $R$ is finite.

The contribution of Theorem 4.4 is that in this symmetric MEU context, No Half Measures is equivalent to limiting the set of measures to the i.i.d. products generated by a finite set of relevant measures, $R$. The proof uses Ghirardato et al. [14, Theorem 14] to show that $F$ is the Bewley set, and then applies Theorems 4.1, 4.2 and 4.3 to obtain the result. Note that the supposition in the first sentence of the theorem could equivalently be replaced by assuming $\succsim$ satisfies Weak Continuous Symmetry and the axioms in Gilboa and Schmeidler [18].

We can apply Theorem 4.4 to the Extended MEU with contraction model to get the following:

Corollary 4.1 Suppose there is a non-constant $v N-M$ utility function $u, a \beta \in[0,1]$, a finite set $D \subseteq \Delta(S)$ and a probability measure $q=\int \ell^{\infty} d m(\ell)$ for an $m \in \Delta(D)$, such that,

$$
\begin{aligned}
V(f) & \equiv \beta \min _{p \in\left\{\ell^{\infty}: \ell \in D\right\}} \int u(f) d p+(1-\beta) \int u(f) d q \\
& =\min _{p \in\left\{\beta \ell^{\infty}+(1-\beta) q: \ell \in D\right\}} \int u(f) d p
\end{aligned}
$$

represents $\succsim$. Then $\succsim$ satisfies No Half Measures if and only if $\left(\beta=1\right.$ or $\left(q \in\left\{\ell^{\infty}: \ell \in D\right\}\right.$ and $(D=\{q\}$ or $\beta=0)))$.

To see that this is a strong condition, observe that the restriction $\left(\beta=1\right.$ or $\left(q \in\left\{\ell^{\infty}: \ell \in D\right\}\right.$ and $(D=\{q\}$ or $\beta=0)))$ is equivalent to MEU without contraction $(\beta=1)$ or expected utility with an i.i.d. prior, ruling out all intermediate, non-extreme, cases of ambiguity attitude.

\section{2 $\alpha$-MEU model}

According to KMS [21, footnote 9] (and the example below), the Bewley set, $C$, generally will depend on $\alpha$ as well as the set of measures appearing in the representation. Since No Half Measures ensures $C$ is fully determined by $R$, it must eliminate the dependence of $C$ on $\alpha$. When does this dependence occur? Our next example (see also Eichberger et al. [8, Section 4] and Eichberger et al. [9, section 3.2.2]), suggests that $\alpha \in(0,1)$ creates dependence.

Example 4.1 Let $S=\{H, T\}$ and denote $\ell \in \Delta(S)$ by the corresponding probability of $H$. Consider the $\succsim$ represented by

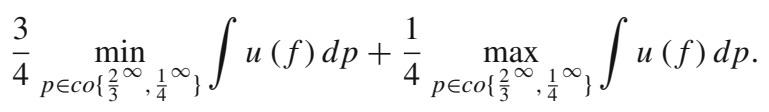

[21, Theorem 4.1] implies that $R=\left\{\frac{2}{3}, \frac{1}{4}\right\}$. Using the results of Siniscalchi ([27]) and a personal communication with him, the Bewley set $C$ is given by $C=\operatorname{co}\left\{\frac{3}{4} \frac{2}{3}^{\infty}+\frac{1}{4} \frac{1}{4}^{\infty}, \frac{1}{4}^{2} \frac{2}{3}^{\infty}+\right.$ $\left.\frac{3}{4} \frac{1}{4}^{\infty}\right\}$, the convex hull of some strict convex combinations of $\left\{\frac{2}{3}^{\infty}, \frac{1}{4}^{\infty}\right\}$ (i.e., convex combinations that are not themselves i.i.d.) and is a proper subset of $c o\left\{\ell^{\infty}: \ell \in R\right\}$ (and, which proper subset is determined by $\alpha=3 / 4$. If, instead, $\alpha=0$ or $1, R$ fully determines $C$ since $C=\operatorname{co}\left\{\frac{2}{3}^{\infty}, \frac{1}{4}^{\infty}\right\}$. In all other cases, when $\alpha \in(0,1)$, the Bewley set $C$ is influenced by $\alpha$. 
Our next result shows that the troublesome case in the example (i.e., $\alpha \in(0,1)$ and $R$ nonsingleton) is exactly what is ruled out by No Half Measures. In this sense, No Half Measures turns out to be extremely restrictive in the context of $\alpha$-MEU:

Theorem 4.5 Suppose there is a non-constant $v N-M$ utility function $u$, an $\alpha \in[0,1]$ and a finite set $D \subseteq \Delta(S)$ such that

$$
V(f) \equiv \alpha \min _{p \in\left\{\ell^{\infty}: \ell \in D\right\}} \int u(f) d p+(1-\alpha) \max _{p \in\left\{\ell^{\infty}: \ell \in D\right\}} \int u(f) d p
$$

represents $\succsim$. Then No Half Measures is equivalent to ( $\alpha=0$ or $\alpha=1$ or D singleton).

Eichberger et al. [9, Theorem 2] prove a related result - for any finite state space, when $C$ is non-singleton, any $\succsim$ having an $\alpha$-MEU representation with Bewley set $C$ as the set of measures has $\alpha$ equal to 0 or 1 . Since $S$ itself, let alone $S^{\infty}$, need not be finite our result requires different arguments. In fact, Eichberger et al. [9] provide an example with an infinite compact metric state space showing that their result may fail in such settings. In light of this, our theorem shows that Event Symmetry and our continuity together with the product structure of the state space is sufficient to extend their finite state space conclusion.

\subsection{Smooth ambiguity model}

Theorem 4.6 Suppose there is a non-constant $v N-M$ utility function $u$, a strictly increasing continuously differentiable function $\phi: u(X) \rightarrow \mathbb{R}$ and a Borel probability measure $\mu \in$ $\Delta(\Delta(S))$ with supp $\mu$ finite such that

$$
U(f) \equiv \int_{\Delta(S)} \phi\left(\int_{S^{\infty}} u(f) d \ell^{\infty}\right) d \mu(\ell)
$$

represents $\succsim$. Then No Half Measures is equivalent to $\left(\sup \bigcup_{r, t \in u(X)} \frac{\phi^{\prime}(t)}{\phi^{\prime}(r)}=+\infty\right.$ or $\operatorname{supp} \mu$ singleton).

Some examples violating the condition $\sup \bigcup_{r, t \in u(X)} \frac{\phi^{\prime}(t)}{\phi^{\prime}(r)}=+\infty$ include $\phi(x)=x$ or $\phi(x)=2 x-\cos (x)$ with $u(X)=\mathbb{R}_{++}$or $\mathbb{R}_{+}$, and $\phi(x)=x+\ln (1+x)$ with $u(X)=$ $\mathbb{R}_{++}$or $\mathbb{R}_{+}$. When utility is bounded both above and below (e.g., there is a best and a worst outcome), it is easy to find examples violating the condition (see Section 4.3 in KMS [21]). Examples satisfying the condition in the theorem include, $\phi(u)=-\exp (-u / \theta)$ or $\phi(x)=x-\exp (-x / \theta)$ with $u(X)=\mathbb{R}$ and $\phi(x)=\frac{x^{1-\gamma}}{1-\gamma}$ or $\phi(x)=x+\frac{x^{1-\gamma}}{1-\gamma}$ for $\gamma \neq 1$ with $u(X)=\mathbb{R}_{++}$or $\mathbb{R}_{+}$.

Following the statement of the No Half Measures condition, we explained that this condition fails when those measures that sometimes get positive weight differ from those that sometimes get arbitrarily close to full weight (where "weight" is measured, as explained earlier, by utility trade-offs between the limiting frequency event corresponding to the measure and its complementary event). By characterizing No Half Measures in the context of the Extended MEU with contraction, $\alpha$-MEU and the smooth ambiguity models, we have seen three concrete illustrations of when the Bewley set, based on (magnitudes of) these positive weights or marginal rates of substitution, will involve parameters usually thought of as tastes (e.g., $\beta, \alpha$ and $\phi$ ) and when it will not. The cases where it will involve these parameters are extensive: For Extended MEU with contraction, No Half Measures fails except for MEU with no contraction or expected utility with an i.i.d. prior. For $\alpha$-MEU, No Half Measures fails in essentially all the cases beyond MEU and max-max EU. For the smooth ambiguity 
model, No Half Measures fails whenever the variation in the slope of $\phi$ is bounded and $\mu$ is non-degenerate.

\section{Appendix: Proofs}

Denote by $B(S)$ the set of bounded measurable functions on $S$. Similarly for $B(\Delta(S))$ and $B\left(S^{\infty}\right)$. When $B$ is replaced by $B_{0}$ this denotes the restriction to simple measurable functions. Similarly, when $B_{0}$ is replaced by $B_{0}^{l}$ (resp. $B_{0}^{u}$ ) this denotes the restriction to lower (resp. upper) semicontinuous simple functions. For $b \in B\left(S^{\infty}\right)$, we write $\|b\|$ for the sup-norm of $b$ (i.e., $\sup _{\omega}|b(\omega)|$ ).

\subsection{Proof of Theorem 3.1}

(i) $\Rightarrow$ (v): Let $f, h \in \mathcal{F}$ and $\pi \in \Pi$. Since $\pi$ is a finite permutation, $\pi^{N}$ is the identity for some $N$. Thus, for any $g \in \mathcal{F}$,

$$
g^{*}(\omega) \equiv \lim _{k \rightarrow \infty} \frac{1}{k} \sum_{i=1}^{k} \pi^{i-1} g(\omega)=\frac{1}{N} \sum_{i=1}^{N} \pi^{i-1} g(\omega)
$$

is well defined everywhere.

Step $1 \mathrm{~g} \sim \mathrm{g}^{*}$.

By repeatedly applying (i),

$$
\begin{aligned}
g & \sim \frac{1}{2} g+\frac{1}{2} \pi g \sim \frac{1}{2}\left(\frac{1}{2} g+\frac{1}{2} \pi g\right)+\frac{1}{2} \pi^{2}\left(\frac{1}{2} g+\frac{1}{2} \pi g\right) \\
& =\frac{1}{2^{2}} \sum_{i=1}^{2^{2}} \pi^{i-1} g \sim \frac{1}{2^{k}} \sum_{i=1}^{2^{k}} \pi^{i-1} g,
\end{aligned}
$$

for all $k$.

For any positive integer $n$, let $q(n)$ be the quotient when we divide $n$ by $N$, and $r(n)$ the remainder. (That is, $n=q(n) \cdot N+r(n)$ ). Then,

$$
\begin{aligned}
\frac{1}{2^{k}} \sum_{i=1}^{2^{k}} \pi^{i-1} g & =\frac{q\left(2^{k}\right)}{2^{k}} \sum_{i=1}^{N} \pi^{i-1} g+\frac{1}{2^{k}} \sum_{i=1}^{r\left(2^{k}\right)} \pi^{i-1} g \\
& =\frac{q\left(2^{k}\right) N}{2^{k}} g^{*}+\frac{1}{2^{k}} \sum_{i=1}^{r\left(2^{k}\right)} \pi^{i-1} g .
\end{aligned}
$$

(Here $\sum_{i=1}^{0} a_{i}$ is understood to be 0 ). Since $r\left(2^{k}\right)$ can take at most finite number of integers, we can find a subsequence $2^{j(k)}$ of $2^{k}$ such that there is $\bar{K}=r\left(2^{j(k)}\right)$ for all $k$. Thus,

$$
g \sim \alpha_{k} g^{*}+\left(1-\alpha_{k}\right)\left(\frac{1}{\bar{K}} \sum_{i=1}^{\bar{K}} \pi^{i-1} g\right) \text { for all } k,
$$

where $\alpha_{k}=\frac{q\left(2^{j(k)}\right) N}{2^{j(k)}}$. (Note that $1-\alpha_{k}=\frac{\bar{K}}{2^{j(k)}}$.) Since $\alpha_{k} \rightarrow 1$, Mixture Continuity implies $g \sim g^{*}$.

Step $2 \alpha f+(1-\alpha) h \sim \alpha \pi f+(1-\alpha) h$. 
By Step 1,

$$
\alpha f+(1-\alpha) h \sim(\alpha f+(1-\alpha) h)^{*}=\alpha f^{*}+(1-\alpha) h^{*} .
$$

Similarly,

$$
\alpha \pi f+(1-\alpha) h \sim \alpha(\pi f)^{*}+(1-\alpha) h^{*}=\alpha f^{*}+(1-\alpha) h^{*} .
$$

By Preorder, this step is proved.

(v) $\Rightarrow$ (iv): By (v),

$$
\alpha f+(1-\alpha) h \sim \alpha \pi f+(1-\alpha) h
$$

for all $\alpha \in[0,1]$ and $h \in \mathcal{F}$. Note that $f \sim \pi f$ when $\alpha=1$.

Setting $h=f$ gives,

$$
f \sim \alpha \pi f+(1-\alpha) f \text { for all } \alpha \in[0,1] .
$$

Moreover, setting $h=\beta f+(1-\beta) \pi^{\prime} f$ leads to

$$
\begin{aligned}
f & \sim(\alpha+(1-\alpha) \beta) f+(1-\alpha)(1-\beta) \pi^{\prime} f(\text { by }(5.2)) \\
& =\alpha f+(1-\alpha)\left(\beta f+(1-\beta) \pi^{\prime} f\right) \\
& \sim \alpha \pi f+(1-\alpha)\left(\beta f+(1-\beta) \pi^{\prime} f\right)(\text { by }(5.1)) \\
& =(1-\alpha) \beta f+\alpha \pi f+(1-\alpha)(1-\beta) \pi^{\prime} f .
\end{aligned}
$$

Since $\alpha$ and $\beta$ can be taken arbitrarily, $f \sim \alpha f+\beta \pi f+(1-\alpha-\beta) \pi^{\prime} f$ for all $\alpha, \beta \in[0,1]$. Since $f \sim \pi^{\prime \prime} f$ for $\pi^{\prime \prime} \in \Pi$,

$$
f \sim \pi^{\prime \prime} f \sim \alpha \pi^{\prime \prime} f+\beta \pi \pi^{\prime \prime} f+(1-\alpha-\beta) \pi^{\prime} \pi^{\prime \prime} f .
$$

For any $\pi_{1}, \pi_{2}, \pi_{3} \in \Pi$, take $\pi=\pi_{2}\left(\pi_{1}\right)^{-1}, \pi^{\prime}=\pi_{3}\left(\pi_{1}\right)^{-1}$ and $\pi^{\prime \prime}=\pi_{1}$. Then, we get (iv) for $n=3$. Repeat the argument to show (iv) for any $n$.

Clearly, (iv) implies (ii) and (iii). Moreover, (ii) implies (i) and so does (iii). Thus, we have shown that (i)-(v) are equivalent.

Assume the additional axioms to show equivalence of (i)-(vii).

(v) $\Leftrightarrow$ (vi): Clearly, (v) implies (vi). By Theorem 4.1, the converse holds.

(vi) $\Leftrightarrow$ (vii): (vi) implies (vii) by Theorem 4.1. The converse holds since

$$
\int(\lambda f+(1-\lambda) h) d p=\int(\lambda \pi f+(1-\lambda) h) d p \text { for all symmetric } p \in \Delta\left(S^{\infty}\right) .
$$

Assume the existence of an extension $\hat{\succsim}$ satisfying Preorder and Norm Continuity to show the equivalence of (i)-(viii).

(vi) $\Leftrightarrow$ (viii): The easy direction is (viii) implies (vi): Note that

$$
(\lambda f+(1-\lambda) h)^{\Psi}=(\lambda \pi f+(1-\lambda) h)^{\Psi}
$$

for all $f, h \in \mathcal{F}$ and $\lambda \in[0,1]$. Thus, (viii) implies $\lambda f+(1-\lambda) h \sim \lambda \pi f+(1-\lambda) h$ for all $f, h \in \mathcal{F}$ and $\lambda \in[0,1]$, which implies Event Symmetry.

We now turn to the other direction. Let $\hat{\succsim}$ be any extension of $\succsim$ to $\hat{\mathcal{F}}$ satisfying Preorder and Norm Continuity. Define $\hat{\succsim}^{*}$ on $\hat{\mathcal{F}}$ by

$$
f \hat{\succsim}^{*} g \text { if } \alpha f+(1-\alpha) h \hat{\succsim} \alpha g+(1-\alpha) h \text { for all } \alpha \in[0,1] \text { and } h \in \hat{\mathcal{F}} \text {. }
$$


Note that $\succsim, \hat{\succsim}, \succsim^{*}$ and $\hat{\succsim}^{*}$ on $X$ all agree and can be represented by some non-constant mixture linear function $u$ on $X$.

Our argument proceeds by steps along the following lines: In steps $1-3$, given an arbitrary $h^{\prime} \in \hat{\mathcal{F}}$, we construct sequences of simple acts that norm-converge to $h^{\prime}$ from above and from below. In steps 4-7, we show that $\hat{\beth}^{*}$ is the unique extension of $\succsim^{*}$ satisfying C-complete Preorder, Norm Continuity, Monotonicity, Independence and Non-triviality. In step 8, we use the representation of $\succsim^{*}$ in Theorem 4.1 to construct a representation for $\hat{\beth}^{*}$, and use that representation to show (vi) implies (viii).

Step 1 For any $h^{\prime} \in \hat{\mathcal{F}}$, and $x, y \in X$ with $x \succ y$, there is $h \in \mathcal{F}$ such that $\frac{1}{2} h(\omega)+$ $\frac{1}{2} y \prec \frac{1}{2} h^{\prime}(\omega)+\frac{1}{2} x$ and $h(\omega) \succsim h^{\prime}(\omega)$ for all $\omega \in \Omega$ : Take $\ldots, x_{-1}, x_{0}, x_{1}, \ldots \in X$ such that $x_{0}=x, x_{-1}=y$ and $u\left(x_{i}\right)-u\left(x_{i-1}\right)=u(x)-u(y)$. For each $\omega \in \Omega$, set $h(\omega)=x_{i}$ if $x_{i-1} \prec h^{\prime}(\omega) \precsim x_{i}$. Notice that $h \in \mathcal{F}$ since $h^{\prime}$ is bounded above and below and $u(x)-u(y)>0$. Then $h$ does the job since $h(\omega) \succsim h^{\prime}(\omega)$ by construction and

$$
\begin{aligned}
\frac{1}{2} u(h(\omega))+\frac{1}{2} u(y) & =\frac{1}{2} u(h(\omega))-\frac{1}{2} u\left(x_{i}\right)+\frac{1}{2} u\left(x_{i-1}\right)+\frac{1}{2} u(x) \\
& <\frac{1}{2} u\left(h^{\prime}(\omega)\right)+\frac{1}{2} u(x) .
\end{aligned}
$$

Step 2 For any $h^{\prime} \in \hat{\mathcal{F}}$, there is $h_{k} \in \mathcal{F}$ such that $h_{k}$ norm-converges to $h^{\prime}$ and $h_{k}(\omega) \succsim h^{\prime}(\omega)$ for all $\omega \in \Omega$ : Take $z_{k}, z \in X$ such that $u\left(z_{k}\right) \searrow u(z)$. This is possible by Mixture Continuity. By Step 1 , for each $k$, since $z_{k} \succ z$, there is $h_{k} \in \mathcal{F}$ such that $h_{k}(\omega) \succsim h^{\prime}(\omega)$ and $\frac{1}{2} h_{k}(\omega)+$ $\frac{1}{2} z \prec \frac{1}{2} h^{\prime}(\omega)+\frac{1}{2} z_{k}$ for all $\omega \in \Omega$. Thus, $\sup _{\omega \in \Omega}\left|u\left(h_{k}(\omega)\right)-u\left(h^{\prime}(\omega)\right)\right| \leq u\left(z_{k}\right)-u(z)$. For any $x>y$, there exists $K$ such that for all $k>K, u\left(z_{k}\right)-u(z) \leq u(x)-u(y)$. Thus, $h_{k}$ norm-converges to $h^{\prime}$.

Step 3 For any $h^{\prime} \in \hat{\mathcal{F}}$, there is $h_{k} \in \mathcal{F}$ such that $h_{k}$ norm-converges to $h^{\prime}$ and $h^{\prime}(\omega) \succsim h_{k}(\omega)$ for all $\omega \in \Omega$ : Slightly change Steps 1 and 2 .

Step $4 \hat{\succsim}$ satisfies Monotonicity: Take $f, g \in \hat{\mathcal{F}}$ such that $f(\omega) \hat{\succsim} g(\omega)$ for all $\omega \in \Omega$. By Steps 2 and 3, there are $f_{k}, g_{k} \in \mathcal{F}$ such that $f_{k}, g_{k}$ norm-converge to $f, g$ respectively, and

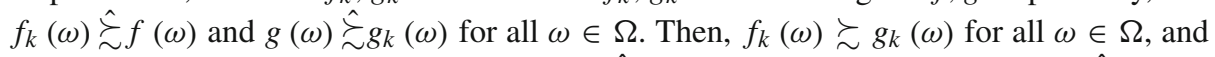
Monotonicity of $\succsim$ implies $f_{k} \succsim g_{k}$, hence $f_{k} \hat{\succsim} g_{k}$. Norm Continuity guarantees $f \hat{\succsim} g$.

Step $5 \hat{\gtrsim}^{*}$ satisfies C-complete Preorder, Norm Continuity, Monotonicity, Independence and Non-triviality: $\hat{\succsim}^{*}$ inherits C-complete Preorder, Norm Continuity, Monotonicity and Nontriviality from the corresponding properties of $\hat{\succsim}$ and satisfies Independence by (5.3).

Step $6 \hat{\succsim}^{*}$ extends $\succsim^{*}$ : Take $f, g \in \mathcal{F}$ such that $f \succsim^{*} g$, that is, $\alpha f+(1-\alpha) h \succsim \alpha g+$ $(1-\alpha) h$ for all $\alpha \in[0,1]$ and $h \in \mathcal{F}$ (and thus $\alpha f+(1-\alpha) h \hat{\succsim} \alpha g+(1-\alpha) h$ for all $\alpha \in[0,1]$ and $h \in \mathcal{F}$ since $\hat{\succsim}$ is an extension of $\succsim)$. Now fix $h^{\prime} \in \hat{\mathcal{F}}$. By Step 2, there is $h_{k} \in \mathcal{F}$ norm-converging to $h^{\prime}$. Moreover, by the mixture linearity of $u, \alpha f+(1-\alpha) h_{k}$ and $\alpha g+(1-\alpha) h_{k}$ norm-converge to $\alpha f+(1-\alpha) h^{\prime}$ and $\alpha g+(1-\alpha) h^{\prime}$ respectively. Since $\alpha f+(1-\alpha) h_{k} \hat{\succsim} \alpha g+(1-\alpha) h_{k}$ for all $\alpha \in[0,1]$ and $k=1,2, \ldots$. Norm Continuity implies $\alpha f+(1-\alpha) h^{\prime} \hat{\succsim} \alpha g+(1-\alpha) h^{\prime}$ for all $\alpha \in[0,1]$. Since $h^{\prime}$ is arbitrary, $f \hat{\succsim}^{*} g$.

Step 7 All extensions of $\succsim^{*}$ satisfying the axioms in Step 5 are the same: Assume $\succsim_{1}^{*}, \succsim_{2}^{*}$ on $\hat{\mathcal{F}}$ are two such extensions. It is enough to show that $f \succsim_{1}^{*} g$ for $f, g \in \hat{\mathcal{F}}$ implies $f \succsim_{2}^{*} g$ since the labeling of the extensions is arbitrary. By Steps 2 and 3, there are $f_{k}, g_{k} \in \mathcal{F}$ such that $f_{k}$ and $g_{k}$ norm-converge to $f$ and $g$ respectively and $f_{k}(\omega) \succsim_{1}^{*} f(\omega), g(\omega) \succsim_{1}^{*} g_{k}(\omega)$ for all $\omega \in \Omega$. Thus, $f_{k} \succsim_{1}^{*} f \succsim_{1}^{*} g \succsim_{1}^{*} g_{k}$. Since $\succsim_{1}^{*}, \succsim_{2}^{*}$ coincide on $\mathcal{F}, f_{k} \succsim_{2}^{*} g_{k}$. Norm Continuity of $\succsim_{2}^{*}$ implies $f \succsim_{2}^{*} g$. 
Step 8 (vi) implies (viii): Theorem 4.1, there is $M \subseteq \Delta(\Delta(S))$ such that for all $f, g \in \mathcal{F}$, $f \succsim^{*} g$ iff $\int u(f) d p \geq \int u(g) d p$ for all $p \in\left\{\int \ell^{\infty} d m(\ell): m \in M\right\}$. Define an extension $\succsim_{1}^{*}$ of $\succsim^{*}$ to $\hat{\mathcal{F}}$ by

$$
f \succsim_{1}^{*} g \text { iff } \int u(f) d p \geq \int u(g) d p \text { for all } p \in\left\{\int \ell^{\infty} d m(\ell): m \in M\right\} .
$$

Then, one can check that $\succsim_{1}^{*}$ satisfies all the axioms in Step 5. By Step 7, $\succsim_{1}^{*}=\hat{\beth}^{*}$. Therefore, $f \hat{\sim}^{*} f^{\Psi}$ and $g \hat{\sim}^{*} g^{\Psi}$ for any $f, g \in \mathcal{F}$ and hence $f \hat{\sim} f^{\Psi}$ and $g \hat{\sim} g \Psi$. Transitivity of $\hat{\succsim}$ implies (viii).

\subsection{Proofs for Sect. 4}

It is sometimes convenient in the proofs to use an alternative formulation of No Half Measures. The following lemma gives the alternative formulation and shows that it is equivalent to No Half Measures. We may rely on this lemma without reference to it.

Lemma 5.1 The following are equivalent:

(a) No Half Measures;

(b) For $A \in \Sigma^{\Psi}$, if there exists an $x \in X$ such that $1_{S^{\infty}} \succ x \succsim^{*} 1_{A}$, then $1_{A} \sim^{*} 1_{\emptyset}$.

Proof of Lemma 5.1 (a) $\Rightarrow$ (b): Fix $A \in \Sigma^{\Psi}$. Suppose for each $x \in X$ with $1_{S^{\infty}} \succ x$, there exists $f \in \mathcal{F}$ and $\alpha \in(0,1]$ such that $\alpha x+(1-\alpha) f \not \alpha 1_{A}+(1-\alpha) f$. Then $x \beth^{*} 1_{A}$ and (b) is vacuously satisfied. Suppose instead that for all $f \in \mathcal{F}$ and $\alpha \in(0,1], \alpha 1_{\emptyset}+(1-\alpha) f \sim$ $\alpha 1_{A}+(1-\alpha) f$. Then $1_{A} \sim^{*} 1_{\emptyset}$ and (b) is satisfied.

(b) $\Rightarrow$ (a): Fix $A \in \Sigma^{\Psi}$. Suppose (b) holds. Can it be that neither of the possibilities in (a) hold? If neither holds, $1_{A} \nsim^{*} 1_{\emptyset}$ and for some $x \in X$ with $1_{S^{\infty}} \succ x$, for all $f \in \mathcal{F}$ and $\alpha \in(0,1], \alpha x+(1-\alpha) f \succsim \alpha 1_{A}+(1-\alpha) f$. But then, $1_{S^{\infty}} \succ x \succsim^{*} 1_{A}$ and $1_{A} \nsim^{*} 1_{\emptyset}$ contradicting (b).

\subsubsection{Proof of Theorem 4.2}

Normalize $u$ so that $u\left(x^{*}\right)=1$ and $u\left(x_{*}\right)=0$.

(if): We show No Half Measures. Fix any $A \in \Sigma^{\Psi}$ and assume $1_{S^{\infty}} \succ x \succsim^{*} 1_{A}$. Then, $u(x) \geq \int u\left(1_{A}\right) d \ell^{\infty}=\ell^{\infty}(A)$ for all $\ell \in R$. Note that $\ell^{\infty}(A) \in\{0,1\}$ for every $A \in \Sigma^{\Psi}$. Since $1_{S^{\infty}} \succ x, 1>u(x)$ and thus $\ell^{\infty}(A)=0$ for all $\ell \in R$. Then, $1_{A} \sim^{*} 0$. By Lemma 5.1, No Half Measures holds.

(only if): Since $\succsim$ satisfies Weak Continuous Symmetry, Theorem 4.1 delivers sets $C, R$ and $M$. By definition, $C \subseteq\left\{\int \ell^{\infty} d m(\ell): m \in \Delta(R)\right\}$. For the other inclusion, follow the steps.

Step 1 For $\widehat{\ell} \in \Delta(S)$, if $\delta_{\widehat{\ell}} \notin M$, then $\max _{m \in M} m(L)<1$ for some $L \in \mathcal{O}_{\widehat{\ell}}$. We show this by contradiction. Suppose that, for every $L \in \mathcal{O}_{\widehat{\ell}}$, there is $m_{L} \in M$ such that $m_{L}(L)=1$. We can view $\mathcal{O}_{\widehat{\ell}}$ as a directed set with the inverse inclusion. Then, $\left\{m_{L}\right\}$ is a net and it has a limit point in $M$ because $M$ is compact. The limit point has to be $\delta_{\widehat{\ell}}$, a contradiction.

Step $2 \widehat{\ell}^{\infty} \in C$ for every $\widehat{\ell} \in R$. To see this, again use proof by contradiction. Suppose not. Then, $\delta_{\widehat{\ell}} \notin M$ for some $\widehat{\ell} \in R$. By Step $1, a=\max _{m \in M} m(L)<1$ for some $L \in \mathcal{O}_{\widehat{\ell}}$. Let $A=\Psi^{-1}(L)$ and $x=a x^{*}+(1-a) x_{*}$. Then, $u(x)=a<1$ and, for any $m \in M$, 


$$
\begin{aligned}
u(x) & =a \geq m(L) \\
& =\int_{L} \ell^{\infty}(A) d m+\int_{\Delta(S) \backslash L} \ell^{\infty}(A) d m\left(\text { since } \ell^{\infty}(A)=1 \text { for } \ell \in L, \text { and } 0 \text { otherwise }\right) \\
& =\int_{L} \int_{S^{\infty}} u\left(1_{A}\right) d \ell^{\infty} d m+\int_{\Delta(S) \backslash L} \int_{S^{\infty}} u\left(1_{A}\right) d \ell^{\infty} d m \\
& =\int_{\Delta(S)} \int_{S^{\infty}} u\left(1_{A}\right) d \ell^{\infty} d m(\ell) .
\end{aligned}
$$

Thus, $1 \succ x \succsim^{*} 1_{A}$. By No Half Measures, $m(L)=\iint u\left(1_{A}\right) d \ell^{\infty} d m(\ell)=0$ for every $m \in M$. But since $\widehat{\ell} \in L$ and $L$ is open, this implies $\widehat{\ell} \notin$ supp $m$ for every $m \in M$ and thus $\hat{\ell} \notin R$. This is a contradiction.

Step $3 C \supseteq\left\{\int \ell^{\infty} d m(\ell): m \in \Delta(R)\right\}$. Denote by $\overline{c o}^{w *}(\cdot)$ the weak* closed convex hull in ba $\left(S^{\infty}\right)$. Observe that

$$
\min _{p \in \overline{c o}^{w *}\left(\left\{\ell^{\infty}: \ell \in R\right\}\right)} \int \psi d p=\inf _{p \in\left\{\ell^{\infty}: \ell \in R\right\}} \int \psi d p=\inf _{p \in\left\{\int \ell^{\infty} d m(\ell): m \in \Delta(R)\right\}} \int \psi d p
$$

for any bounded measurable $\psi$ on $S^{\infty}$. Since the set of measures in the MEU functional is unique up to the weak* closed convex hull (Gilboa and Schmeidler [18]),

$$
\overline{c o}^{w *}\left(\left\{\ell^{\infty}: \ell \in R\right\}\right) \supseteq\left\{\int \ell^{\infty} d m(\ell): m \in \Delta(R)\right\} .
$$

By this inclusion, Step 2 and weak* closedness and convexity of $C$, we have

$$
C \supseteq \overline{c o}^{w *}\left(\left\{\ell^{\infty}: \ell \in R\right\}\right) \supseteq\left\{\int \ell^{\infty} d m(\ell): m \in \Delta(R)\right\} .
$$

This completes the proof.

\subsubsection{Proof of Theorem 4.3}

Suppose $R$ is not finite. Then, we can take distinct $\ell_{n} \in R$ for each $n$. Let $A_{n}=$ $\bigcup_{k \geq n} \Psi^{-1}\left(\ell_{k}\right)$. Then, $A_{n} \searrow \emptyset$. To try to verify Monotone Continuity of $\succsim^{*}$, take three constant acts $x \succ x^{\prime} \succ x^{\prime \prime}$. Let $p_{n}=\ell_{n}^{\infty}$. Then, $p_{n}\left(A_{n}\right)=1$ and $u\left(x^{\prime}\right)<p_{n}\left(A_{n}\right) u(x)+$ $\left(1-p_{n}\left(A_{n}\right)\right) u\left(x^{\prime \prime}\right)$. By Theorem 4.2, $p_{n} \in C$ and Theorem 4.1 implies that it is not possible that $x^{\prime} \succsim^{*} x A_{n} x^{\prime \prime}$ for any $n$. This contradicts Monotone Continuity of $\succsim^{*}$.

\subsubsection{Proof of Theorem 4.4}

Fix the representation $V$.

(if) If $\succsim$ is represented by $\min _{\ell \in R} \int_{S^{\infty}} u(f) d \ell^{\infty}$ then the fact that $\succsim$ satisfies No Half Measures follows from one direction of Theorem 4.5.

(only if) By Ghirardato et al. [14, Theorem 14], the Bewley set, $C$, associated with $\succsim$ is $F$. By Theorem 4.1, $\succsim$ satisfies Weak Continuous Symmetry. Suppose No Half Measures. Then, by Theorems 4.2 and 4.3, $C=\left\{\int \ell^{\infty} d m(\ell): m \in \Delta(R)\right\}$ and $R$ is finite. Thus,

$$
V(f)=\min _{p \in F} \int u(f) d p=\min _{p \in\left\{\int \ell^{\infty} d m(\ell): m \in \Delta(R)\right\}} \int u(f) d p=\min _{\ell \in R} \int_{S^{\infty}} u(f) d \ell^{\infty} .
$$




\subsubsection{Proof of Theorem 4.5}

We first show ( $\alpha=0$ or $\alpha=1$ or $D$ singleton) implies No Half Measures. Recall that No Half Measures concerns only events $A \in \Sigma^{\Psi}$. If $D$ singleton, then $1_{S^{\infty}} \succ x \succsim 1_{A}$ implies $\ell^{\infty}(A)=0$ for the $\ell \in D$, in which case $1_{A} \sim^{*} 1_{\emptyset}$ is obvious. To see the other cases, observe that $1_{S^{\infty}} \succ x$ if and only if $u(x)<1$ and $x \succsim^{*} 1_{A}$ if and only if

$$
\begin{aligned}
& \lambda u(x)+(1-\lambda)\left[\alpha \min _{p \in\left\{\ell^{\infty}: \ell \in D\right\}} \int u(h) d p+(1-\alpha) \max _{p \in\left\{\ell^{\infty}: \ell \in D\right\}} \int u(h) d p\right] \\
& \geq \alpha \min _{p \in\left\{\ell^{\infty}: \ell \in D\right\}}\left(\lambda p(A)+(1-\lambda) \int u(h) d p\right)+(1-\alpha) \\
& \quad \times \max _{p \in\left\{\ell^{\infty}: \ell \in D\right\}}\left(\lambda p(A)+(1-\lambda) \int u(h) d p\right)
\end{aligned}
$$

for all $\lambda \in[0,1]$ and all acts $h$. Furthermore, $1_{A} \sim^{*} 1_{\emptyset}$ if and only if

$$
\begin{aligned}
& \alpha \min _{p \in\left\{\ell^{\infty}: \ell \in D\right\}}\left(\lambda p(A)+(1-\lambda) \int u(h) d p\right) \\
& \quad+(1-\alpha) \max _{p \in\left\{\ell^{\infty}: \ell \in D\right\}}\left(\lambda p(A)+(1-\lambda) \int u(h) d p\right) \\
& \quad=\alpha \min _{p \in\left\{\ell^{\infty}: \ell \in D\right\}}\left((1-\lambda) \int u(h) d p\right)+(1-\alpha) \max _{p \in\left\{\ell^{\infty}: \ell \in D\right\}}\left((1-\lambda) \int u(h) d p\right)
\end{aligned}
$$

for all $\lambda \in[0,1]$ and all acts $h$. In fact, $1_{A} \sim^{*} 1_{\varnothing}$ if and only if $p(A)=0$ for all $p \in$ $\left\{\ell^{\infty}: \ell \in D\right\}$. To see this last statement, note that the "if" direction is immediate, the "only if" direction follows for $\alpha \neq 1$ by setting $\lambda=1$ in (5.5), and if $\alpha=1$, it follows by setting $\lambda=\frac{1}{3}$ and $h=1_{A^{c}}$ in (5.5). Therefore, in the presence of the other requirements in the theorem, No Half Measures is equivalent to the statement: For all $A \in \Sigma^{\Psi}, p(A)=1$ for some $p \in\left\{\ell^{\infty}: \ell \in D\right\}$ implies there does not exist an $x \in X$ such that $u(x)<1$ and (5.4) satisfied. Now consider the case $\alpha=0$ and let $h=1_{A}$. Then the right-hand side of (5.4) becomes 1 and therefore greater than any $u(x)<1$ violating (5.4). Finally consider $\alpha=1$ and let $\lambda=\frac{1}{3}$ and $h=1_{A^{c}}$ in (5.4). Simplifying yields

$$
\frac{1}{3} u(x)+\frac{2}{3}\left[\min _{p \in\left\{\ell^{\infty}: \ell \in D\right\}}(1-p(A))\right] \geq \min _{p \in\left\{\ell^{\infty}: \ell \in D\right\}}\left(\frac{1}{3} p(A)+\frac{2}{3}(1-p(A))\right)
$$

which simplifies to $u(x) \geq 1$, thus showing No Half Measures holds.

We next show the other direction-that No Half Measures implies ( $\alpha=0$ or $\alpha=1$ or $D$ singleton). To see this, we assume $D$ non-singleton and $\alpha \in(0,1)$ and show that No Half Measures is violated.

No Half Measures violated is equivalent, by the arguments above, to, for some $A \in \Sigma^{\Psi}$, $p(A)=1$ for some $p \in\left\{\ell^{\infty}: \ell \in D\right\}$ while (5.4) is satisfied for some $u(x)<1$. To this end, consider $A=\Psi^{-1}(\hat{\ell})$ for some $\hat{p}=(\hat{\ell})^{\infty} \in\left\{\ell^{\infty}: \ell \in D\right\}$. Observe that $\hat{p}(A)=1$, $p(A)=0$ for all $p \in\left\{\ell^{\infty}: \ell \in D\right\} \backslash\{\hat{p}\}$ and $\left\{\ell^{\infty}: \ell \in D\right\} \backslash\{\hat{p}\}$ is non-empty (since $D$ non-singleton). For this event $A$, we will show that (5.4) is satisfied for $u(x)=\frac{1+\max [\alpha, 1-\alpha]}{2}$. Note that $\alpha \in(0,1)$ implies $\max [\alpha, 1-\alpha]<u(x)<1$. Rewriting (5.4) using this $A$ and $u(x)$, we want to show 


$$
\begin{aligned}
& \alpha \min \left[\lambda+(1-\lambda) \int u(h) d \hat{p}, \min _{p \in\left\{\ell^{\infty}: \ell \in D\right\} \backslash\{\hat{p}\}}(1-\lambda) \int u(h) d p\right] \\
& +(1-\alpha) \max \left[\lambda+(1-\lambda) \int u(h) d \hat{p}, \max _{p \in\left\{\ell^{\infty}: \ell \in D\right\} \backslash\{\hat{p}\}}(1-\lambda) \int u(h) d p\right] \\
& \leq \lambda \frac{1+\max [\alpha, 1-\alpha]}{2}+(1-\lambda)\left[\alpha \min _{p \in\left\{\ell^{\infty}: \ell \in D\right\}} \int u(h) d p+(1-\alpha) \max _{p \in\left\{\ell^{\infty}: \ell \in D\right\}} \int u(h) d p\right]
\end{aligned}
$$

holds for all acts $h$ and all $\lambda \in[0,1]$. If $\lambda=0$, (5.6) holds with equality. If $\lambda=1$, (5.6) simplifies to $1-\alpha \leq \frac{1+\max [\alpha, 1-\alpha]}{2}$, which is true. For the remainder of the argument we assume $\lambda \in(0,1)$. We proceed by considering three mutually exclusive and exhaustive cases: (1) $\hat{p} \notin \arg \min _{p \in\left\{\ell^{\infty}: \ell \in D\right\}} \int u(h) d p$, (2) $\hat{p} \in \arg \min _{p \in\left\{\ell^{\infty}: \ell \in D\right\}} \int u(h) d p$ and $\frac{\lambda}{1-\lambda}+\min _{p \in\left\{\ell^{\infty}: \ell \in D\right\}} \int u(h) d p \leq \max _{p \in\left\{\ell^{\infty}: \ell \in D\right\}} \int u(h) d p$ and $(3) \hat{p} \in \arg \min _{p \in\left\{\ell^{\infty}: \ell \in D\right\}}$ $\int u(h) d p$ and $\left.\frac{\lambda}{1-\lambda}+\min _{p \in\{\ell \infty}: \ell \in D\right\} \int u(h) d p>\max _{p \in\left\{\ell^{\infty}: \ell \in D\right\}} \int u(h) d p$. For each case we show (5.6) holds.

Case (1) If $\hat{p} \notin \arg \min _{p \in\left\{\ell^{\infty}: \ell \in D\right\}} \int u(h) d p$, then $p(A)$ and $\int u(h) d p$ have a common minimizer located in $\left\{\ell^{\infty}: \ell \in D\right\} \backslash\{\hat{p}\}$ and

$$
\begin{aligned}
\alpha & \min \left[\lambda+(1-\lambda) \int u(h) d \hat{p}, \min _{p \in\left\{\ell^{\infty}: \ell \in D\right\} \backslash\{\hat{p}\}}(1-\lambda) \int u(h) d p\right] \\
& +(1-\alpha) \max \left[\lambda+(1-\lambda) \int u(h) d \hat{p}, \max _{p \in\left\{\ell^{\infty}: \ell \in D\right\} \backslash\{\hat{p}\}}(1-\lambda) \int u(h) d p\right] \\
= & \alpha(1-\lambda) \min _{p \in\left\{\ell^{\infty}: \ell \in D\right\}} \int u(h) d p+(1-\alpha) \max _{p \in\left\{\ell^{\infty}: \ell \in D\right\}}\left(\lambda p(A)+(1-\lambda) \int u(h) d p\right) \\
\leq & \lambda(1-\alpha)+(1-\lambda)\left[\alpha \min _{p \in\left\{\ell^{\infty}: \ell \in D\right\}} \int u(h) d p+(1-\alpha) \max _{p \in\left\{\ell^{\infty}: \ell \in D\right\}} \int u(h) d p\right] \\
< & \lambda \frac{1+\max [\alpha, 1-\alpha]}{2} \\
& +(1-\lambda)\left[\alpha \min _{p \in\left\{\ell^{\infty}: \ell \in D\right\}} \int u(h) d p+(1-\alpha) \max _{p \in\left\{\ell^{\infty}: \ell \in D\right\}} \int u(h) d p\right] .
\end{aligned}
$$

Case (2) If $\hat{p} \in \arg \min _{p \in\left\{\ell^{\infty}: \ell \in D\right\}} \int u(h) d p$ and $\frac{\lambda}{1-\lambda}+\min _{p \in\left\{\ell^{\infty}: \ell \in D\right\}} \int u(h) d p \leq$ $\max _{p \in\left\{\ell^{\infty}: \ell \in D\right\}} \int u(h) d p$, then, noting that

$$
\max _{p \in\left\{\ell^{\infty}: \ell \in D\right\}} \int u(h) d p=\max _{p \in\left\{\ell^{\infty}: \ell \in D\right\} \backslash\{\hat{p}\}} \int u(h) d p,
$$

we have

$$
\begin{aligned}
& \alpha \min \left[\lambda+(1-\lambda) \int u(h) d \hat{p}, \min _{p \in\left\{\ell^{\infty}: \ell \in D\right\} \backslash\{\hat{p}\}}(1-\lambda) \int u(h) d p\right] \\
& +(1-\alpha) \max \left[\lambda+(1-\lambda) \int u(h) d \hat{p}, \max _{p \in\left\{\ell^{\infty}: \ell \in D\right\} \backslash\{\hat{p}\}}(1-\lambda) \int u(h) d p\right] \\
& =\alpha \min \left[\lambda+(1-\lambda) \int u(h) d \hat{p}, \min _{p \in\left\{\ell^{\infty}: \ell \in D\right\} \backslash\{\hat{p}\}}(1-\lambda) \int u(h) d p\right] \\
& \quad+(1-\alpha)(1-\lambda) \max _{p \in\left\{\ell^{\infty}: \ell \in D\right\}} \int u(h) d p \\
& \leq \lambda \alpha+(1-\lambda)\left[\alpha \int u(h) d \hat{p}+(1-\alpha) \max _{p \in\left\{\ell^{\infty}: \ell \in D\right\}} \int u(h) d p\right]
\end{aligned}
$$




$$
\begin{aligned}
& <\lambda \frac{1+\max [\alpha, 1-\alpha]}{2} \\
& +(1-\lambda)\left[\alpha \min _{p \in\left\{\ell^{\infty}: \ell \in D\right\}} \int u(h) d p+(1-\alpha) \max _{p \in\left\{\ell^{\infty}: \ell \in D\right\}} \int u(h) d p\right] .
\end{aligned}
$$

Case (3) If $\hat{p} \in \arg \min _{p \in\left\{\ell^{\infty}: \ell \in D\right\}} \int u(h) d p$ and $\frac{\lambda}{1-\lambda}+\min _{p \in\left\{\ell^{\infty}: \ell \in D\right\}} \int u(h) d p>$ $\max _{p \in\left\{\ell^{\infty}: \ell \in D\right\}} \int u(h) d p$, then

$$
\begin{aligned}
& \alpha \min \left[\lambda+(1-\lambda) \int u(h) d \hat{p}, \min _{p \in\left\{\ell^{\infty}: \ell \in D\right\} \backslash\{\hat{p}\}}(1-\lambda) \int u(h) d p\right] \\
& +(1-\alpha) \max \left[\lambda+(1-\lambda) \int u(h) d \hat{p}, \max _{p \in\left\{\ell^{\infty}: \ell \in D\right\} \backslash\{\hat{p}\}}(1-\lambda) \int u(h) d p\right] \\
& =\alpha(1-\lambda) \min _{p \in\left\{\ell^{\infty}: \ell \in D\right\} \backslash\{\hat{p}\}} \int u(h) d p+(1-\alpha)\left(\lambda+(1-\lambda) \int u(h) d \hat{p}\right) \\
& =\lambda(1-\alpha)+(1-\lambda)\left[\alpha \left(\min _{p \in\left\{\ell^{\infty}: \ell \in D\right\} \backslash\{\hat{p}\}} \int u(h) d p\right.\right. \\
& \left.+\min _{p \in\left\{\ell^{\infty}: \ell \in D\right\}} \int u(h) d p-\min _{p \in\left\{\ell^{\infty}: \ell \in D\right\}} \int u(h) d p\right) \\
& +(1-\alpha)\left(\min _{p \in\left\{\ell^{\infty}: \ell \in D\right\}} \int u(h) d p\right. \\
& \left.\left.+\max _{p \in\left\{\ell^{\infty}: \ell \in D\right\}} \int u(h) d p-\max _{p \in\left\{\ell^{\infty}: \ell \in D\right\}} \int u(h) d p\right)\right] \\
& =\lambda(1-\alpha) \\
& +(1-\lambda)\left[\alpha \min _{p \in\left\{\ell^{\infty}: \ell \in D\right\}} \int u(h) d p+(1-\alpha) \max _{p \in\left\{\ell^{\infty}: \ell \in D\right\}} \int u(h) d p\right] \\
& +(1-\lambda)\left[(\alpha-1)\left(\max _{p \in\left\{\ell^{\infty}: \ell \in D\right\}} \int u(h) d p-\min _{p \in\left\{\ell^{\infty}: \ell \in D\right\}} \int u(h) d p\right)\right. \\
& \left.+\alpha\left(\min _{p \in\left\{\ell^{\infty}: \ell \in D \backslash\{\hat{p}\}\right\}} \int u(h) d p-\min _{p \in\left\{\ell^{\infty}: \ell \in D\right\}} \int u(h) d p\right)\right] \\
& \leq \lambda(1-\alpha)+(1-\lambda)\left[\alpha \min _{p \in\left\{\ell^{\infty}: \ell \in D\right\}} \int u(h) d p+(1-\alpha) \max _{p \in\left\{\ell^{\infty}: \ell \in D\right\}} \int u(h) d p\right] \\
& +(1-\lambda)(2 \alpha-1)\left(\max _{p \in\left\{\ell^{\infty}: \ell \in D\right\}} \int u(h) d p-\min _{p \in\left\{\ell^{\infty}: \ell \in D\right\}} \int u(h) d p\right) \\
& =\lambda \frac{1+\max [\alpha, 1-\alpha]}{2} \\
& +(1-\lambda)\left[\alpha \min _{p \in\left\{\ell^{\infty}: \ell \in D\right\}} \int u(h) d p+(1-\alpha) \max _{p \in\left\{\ell^{\infty}: \ell \in D\right\}} \int u(h) d p\right] \\
& +\lambda\left(1-\alpha-\frac{1+\max [\alpha, 1-\alpha]}{2}\right) \\
& +(1-\lambda)(2 \alpha-1)\left(\max _{p \in\left\{\ell^{\infty}: \ell \in D\right\}} \int u(h) d p-\min _{p \in\left\{\ell^{\infty}: \ell \in D\right\}} \int u(h) d p\right) .
\end{aligned}
$$

To complete Case (3), we must show that the last two terms in the final expression above have a non-positive sum. Observe that the hypothesis of Case (3) implies 


$$
0 \leq\left(\max _{p \in\left\{\ell^{\infty}: \ell \in D\right\}} \int u(h) d p-\min _{p \in\left\{\ell^{\infty}: \ell \in D\right\}} \int u(h) d p\right)<\frac{\lambda}{1-\lambda} .
$$

Since $\left(\max _{p \in\left\{\ell^{\infty}: \ell \in D\right\}} \int u(h) d p-\min _{p \in\left\{\ell^{\infty}: \ell \in D\right\}} \int u(h) d p\right)$ enters linearly, the sum of the last two terms is bounded above by the maximum of the sum when substituting 0 for $\left(\max _{p \in\left\{\ell^{\infty}: \ell \in D\right\}} \int u(h) d p-\min _{p \in\left\{\ell^{\infty}: \ell \in D\right\}} \int u(h) d p\right)$ and the sum when substituting $\frac{\lambda}{1-\lambda}$. Carrying out these substitutions and simplifying yields

$$
\begin{aligned}
& \lambda\left(1-\alpha-\frac{1+\max [\alpha, 1-\alpha]}{2}\right) \\
& \quad+(1-\lambda)(2 \alpha-1)\left(\max _{p \in\left\{\ell^{\infty}: \ell \in D\right\}} \int u(h) d p-\min _{p \in\left\{\ell^{\infty}: \ell \in D\right\}} \int u(h) d p\right) \\
& \quad \leq \max \left[\lambda\left(1-\alpha-\frac{1+\max [\alpha, 1-\alpha]}{2}\right), \lambda\left(\alpha-\frac{1+\max [\alpha, 1-\alpha]}{2}\right)\right] \\
& \quad=\lambda\left(\frac{\max [\alpha, 1-\alpha]-1}{2}\right)<0 .
\end{aligned}
$$

\subsubsection{Proof of Theorem 4.6}

Normalize $u\left(x^{*}\right)=1$ and $u\left(x_{*}\right)=0$.

(if): Suppose $\sup \bigcup_{r, t \in u(X)} \frac{\phi^{\prime}(t)}{\phi^{\prime}(r)}=+\infty$. No Half Measures says that for $A \in \Sigma^{\Psi}$, if there exists an $x \in X$ such that $1_{S^{\infty}} \succ x \succsim^{*} 1_{A}$, then $1_{A} \sim^{*} 1_{\emptyset}$. We prove this by showing that, for $A \in \Sigma^{\Psi}, 1_{A} \nsim^{*} 1_{\emptyset}$ implies there does not exist an $x \in X$ such that $1_{S^{\infty}} \succ x \succsim^{*} 1_{A}$. Suppose $1_{A} \nsim^{*} 1_{\emptyset}$. In terms of the representation, using the definition of $\succsim^{*}$, this nonindifference may be written as follows:

$$
\int_{\Delta(S)} \phi\left(\lambda \ell^{\infty}(A)+(1-\lambda) \int_{S^{\infty}} u(h) d \ell^{\infty}\right) d \mu(\ell) \neq \int_{\Delta(S)} \phi\left((1-\lambda) \int_{S^{\infty}} u(h) d \ell^{\infty}\right) d \mu(\ell)
$$

for some $\lambda \in[0,1]$ and act $h$. From the facts that $\phi$ is strictly increasing and $\ell^{\infty}(A) \in\{0,1\}$, this is equivalent to the requirement that $\mu(B)>0$ for the set $B \equiv\left\{\ell \in \Delta(S): \ell^{\infty}(A)=1\right\}$. Now we show that $1_{S^{\infty}} \succ x \succsim^{*} 1_{A}$ is impossible. Observe that $1_{S^{\infty}} \succ x$ means simply $u(x)<1$. Similarly, $x \succsim^{*} 1_{A}$ if and only if

$$
\begin{aligned}
& \int_{\Delta(S)} \phi\left(\lambda u(x)+(1-\lambda) \int_{S^{\infty}} u(h) d \ell^{\infty}\right) d \mu(\ell) \\
& \geq \int_{\Delta(S)} \phi\left(\lambda \ell^{\infty}(A)+(1-\lambda) \int_{S^{\infty}} u(h) d \ell^{\infty}\right) d \mu(\ell)
\end{aligned}
$$

for all $\lambda \in[0,1]$ and all acts $h$. Notice that when $\lambda=0,(5.7)$ is automatically satisfied with equality. Therefore a necessary condition for $x \succsim^{*} 1_{A}$ to hold is that when both sides of (5.7) are differentiated with respect to $\lambda$ and evaluated at $\lambda=0$ the derivative on the left-hand side is weakly larger than that on the right-hand side. Doing this differentiation and evaluating at $\lambda=0$ yields 


$$
\begin{aligned}
& \int_{\Delta(S)}\left(u(x)-\int_{S^{\infty}} u(h) d \ell^{\infty}\right) \phi^{\prime}\left(\int_{S^{\infty}} u(h) d \ell^{\infty}\right) d \mu(\ell) \\
& \geq \int_{\Delta(S)}\left(\ell^{\infty}(A)-\int_{S^{\infty}} u(h) d \ell^{\infty}\right) \phi^{\prime}\left(\int_{S^{\infty}} u(h) d \ell^{\infty}\right) d \mu(\ell),
\end{aligned}
$$

which simplifies to

$$
u(x) \int_{\Delta(S)} \phi^{\prime}\left(\int_{S^{\infty}} u(h) d \ell^{\infty}\right) d \mu(\ell) \geq \int_{\Delta(S)} \ell^{\infty}(A) \phi^{\prime}\left(\int_{S^{\infty}} u(h) d \ell^{\infty}\right) d \mu(\ell) .
$$

Consider acts $h$ that are bets on $A$ (or $A^{c}$ ) such that $u(h)=a_{A} b$ for $a, b \in u(X)$. Such acts exist for any $a, b \in u(X)$. For such an $h,(5.8)$ simplifies to

$$
u(x)\left[\phi^{\prime}(a) \mu(B)+\phi^{\prime}(b)(1-\mu(B))\right] \geq \phi^{\prime}(a) \mu(B) .
$$

Since $\mu(B)>0$ and $\sup \bigcup_{r, t \in u(X)} \frac{\phi^{\prime}(t)}{\phi^{\prime}(r)}=+\infty$, we can choose $a$ and $b$ to make $\frac{\phi^{\prime}(a)}{\phi^{\prime}(b)}$ as large as desired, leading the right-hand side of (5.9) to be as close as desired to 1 and violating the inequality (since $u(x)<1$ ). This completes the "if" direction of the proof.

(only if): We now show the converse under the assumption that $\operatorname{supp} \mu$ is non-singleton (if $\operatorname{supp} \mu$ is a singleton, then properties of $\phi$ beyond the fact that it is strictly increasing cannot affect preferences). By the proof of KMS [21, Theorem 4.3] and the fact that supp $\mu$ is finite, $\succsim$ satisfies Symmetry, Mixture Continuity of ${ }^{*}$ and Monotone Continuity of $\succsim^{*}$. Suppose No Half Measures holds. By Theorem 4.2, $C=\left\{\int \ell^{\infty} d m(\ell): m \in \Delta(R)\right\}$. By KMS [21, Theorem 4.3], $R=\operatorname{supp} \mu$. Therefore $C=\left\{\int \ell^{\infty} d m(\ell): m \in \Delta(\operatorname{supp} \mu)\right\}$. Fix any $\ell_{1} \in \operatorname{supp} \mu$. Note that $\ell_{1}^{\infty} \in C$ and is an extreme point of $C$. By the characterization of $C$ from Proposition 17 of Ghirardato and Siniscalchi [15], simplified using the i.i.d. structure and the continuous differentiability of $\phi$,

$$
C=\overline{c o}\left(\left\{\frac{\int \phi^{\prime}\left(\int e d \ell^{\infty}\right) \ell^{\infty} d \mu(\ell)}{\int \phi^{\prime}\left(\int e d \ell^{\infty}\right) d \mu(\ell)}: \ell \in \Delta(S), e \in \operatorname{int} B_{b}(\Sigma, u(X))\right\}\right),
$$

where int $B_{b}(\Sigma, u(X))$ is the interior of the set of all $\Sigma$-measurable functions $a$ : $S^{\infty} \rightarrow \mathbb{R}$ for which there exist $\alpha, \beta \in u(X)$ satisfying $\alpha \geq a(\omega) \geq \beta$ for all $\omega \in S^{\infty}$ (i.e., informally, the interior of the set of bounded utility-acts). Since $\ell_{1}^{\infty} \in C$ and is an extreme point of $C$, it must be that there exists a sequence of measures in $\left\{\frac{\int \phi^{\prime}\left(\int e d \ell^{\infty}\right) \ell^{\infty} d \mu(\ell)}{\int \phi^{\prime}\left(\int e d \ell^{\infty}\right) d \mu(\ell)}: \ell \in \Delta(S), e \in\right.$ int $\left.B_{b}(\Sigma, u(X))\right\}$ converging to $\ell_{1}^{\infty}$. If $L \subseteq \Delta(S)$ is an open set containing $\ell_{1}, \mu(L)>0$. Since supp $\mu$ is non-singleton, there exist open sets containing $\ell_{1}$ such that $\mu(L)<1$. Let $\hat{L}$ be such a set and consider the event $A=\Psi^{-1}(\hat{L})$. Suppose $\sup \bigcup_{r, t \in u(X)} \frac{\phi^{\prime}(t)}{\phi^{\prime}(r)}=K<+\infty$. Then

$$
\begin{aligned}
& \sup \left\{\frac{\int \phi^{\prime}\left(\int e d \ell^{\infty}\right) \ell^{\infty}(A) d \mu(\ell)}{\int \phi^{\prime}\left(\int e d \ell^{\infty}\right) d \mu(\ell)}: \ell \in \Delta(S), e \in \operatorname{int} B_{b}(\Sigma, u(X))\right\} \\
& =\sup \left\{\frac{\int_{\hat{L}} \phi^{\prime}\left(\int e d \ell^{\infty}\right) d \mu(\ell)}{\int_{\hat{L}} \phi^{\prime}\left(\int e d \ell^{\infty}\right) d \mu(\ell)+\int_{\Delta(S) \backslash \hat{L}} \phi^{\prime}\left(\int e d \ell^{\infty}\right) d \mu(\ell)}: \ell \in \Delta(S), e \in \operatorname{int} B_{b}(\Sigma, u(X))\right\} \\
& \leq \frac{K}{K+1}<1=\ell_{1}^{\infty}(A)
\end{aligned}
$$


so that no sequence in $\left\{\frac{\int \phi^{\prime}\left(\int e d \ell^{\infty}\right) \ell^{\infty}(A) d \mu(\ell)}{\int \phi^{\prime}\left(\int e d \ell^{\infty}\right) d \mu(\ell)}: \ell \in \Delta(S), e \in\right.$ int $\left.B_{b}(\Sigma, u(X))\right\}$ can converge to $\ell_{1}^{\infty}$, a contradiction. Thus, $\sup \bigcup_{r, t \in u(X)} \frac{\phi^{\prime}(t)}{\phi^{\prime}(r)}=+\infty$, completing the proof.

Open Access This article is distributed under the terms of the Creative Commons Attribution 4.0 International License (http://creativecommons.org/licenses/by/4.0/), which permits unrestricted use, distribution, and reproduction in any medium, provided you give appropriate credit to the original author(s) and the source, provide a link to the Creative Commons license, and indicate if changes were made.

\section{References}

1. Aliprantis, C.D., Border, K.C.: Infinite Dimensional Analysis, 3rd edn. Springer, Berlin (2006)

2. Al-Najjar, N.I., de Castro, L.: Parametric representation of preferences. J. Econ. Theory 115, 118-133 (2014)

3. Arrow, K.: Essays in the Theory of Risk-Bearing, North-Holland (1970)

4. Bewley, T.F.: Knightian decision theory, Part I, Decisions in Economics and Finance 25 (2002), 79-110, first version 1986

5. de Castro, L., Al-Najjar, N.I.: A subjective foundation of objective probability, working paper (2009)

6. Cerreia-Vioglio, S., Maccheroni, F., Marinacci, M., Montrucchio, L.: Ambiguity and robust statistics. J. Econ. Theory 148, 974-1049 (2013)

7. de Finetti, B.: La prevision: ses lois logiques, ses sources subjectives. Annales de l'Institut Henri Poincare 7, 1-68 (1937)

8. Eichberger, J., Grant, S., Kelsey, D.: Differentiating ambiguity: an expository note. Econ. Theory 36, 327-336 (2008)

9. Eichberger, J., Grant, S., Kelsey, D., Koshevoy, G.: The $\alpha$-MEU model: a comment. J. Econ. Theory 146, 1684-1698 (2011)

10. Epstein, L.G., Seo, K.: Symmetry of evidence without evidence of symmetry. Theor. Econ. 5, 313-368 (2010)

11. Epstein, L.G., Seo, K.: Symmetry or dynamic consistency?. B.E. J. Theor. Econ. 11, Article 11 (2011)

12. Epstein, L.G., Seo, K.: De Finetti meets Ellsberg. Res. Econ. 68, 11-26 (2014)

13. Gajdos, T., Hayashi, T., Tallon, J.-M., Vergnaud, J.-C.: Attitude toward imprecise information. J. Econ. Theory 140, 23-56 (2008)

14. Ghirardato, P., Maccheroni, F., Marinacci, M.: Differentiating ambiguity and ambiguity attitude. J. Econ. Theory 118, 133-173 (2004)

15. Ghirardato, P., Siniscalchi, M.: A more robust definition of multiple priors, working paper (2010)

16. Ghirardato, P., Siniscalchi, M.: Ambiguity in the small and in the large. Econometrica 80, 2827-2847 (2012)

17. Gilboa, I., Marinacci, M.: Ambiguity and the Bayesian paradigm, advances in economics and econometrics: theory and applications. In: Acemoglu D., Arellano M., Dekel E. (eds.) Tenth World Congress of the Econometric Society. New York: Cambridge University Press (2013)

18. Gilboa, I., Schmeidler, D.: Maxmin expected utility with non-unique prior. J. Math. Econ. 18, 141-153 (1989)

19. Hewitt, E., Savage, L.J.: Symmetric measures on Cartesian products. Trans. Am. Math. Soc. 80, 470-501 (1955)

20. Klibanoff, P., Marinacci, M., Mukerji, S.: A smooth model of decision making under ambiguity. Econometrica 73, 1849-1892 (2005)

21. Klibanoff, P., Mukerji, S., Seo, K.: Perceived ambiguity and relevant measures. Econometrica 82, 19451978 (2014)

22. Marinacci, M.: Model Uncertainty. J. Euro. Econ. Assoc. 13, 998-1076 (2015)

23. Nau, R.: Uncertainty aversion with second-order utilities and probabilities. Manag. Sci. 52, 136-145 (2006)

24. Nehring, K.: Ambiguity in the context of probabilistic beliefs, working paper (2001)

25. Nehring, K.: Bernoulli without Bayes: a theory of utility-sophisticated preference, working paper (2007)

26. Seo, K.: Ambiguity and second-order belief. Econometrica 77, 1575-1605 (2009)

27. Siniscalchi, M.: A behavioral characterization of plausible priors. J. Econ. Theory 128, 91-135 (2006)

28. Siniscalchi, M.: Vector expected utility and attitudes toward variation. Econometrica 77, 801-855 (2009) 